\title{
Development and Validation of a Clinically Relevant Workflow for MR-Guided Volumetric Arc Therapy in a Rabbit Model of Head and Neck Cancer
}

\author{
Eftekhar Rajab Bolookat ${ }^{1,2}$, Harish Malhotra ${ }^{2,3}$, Laurie J. Rich ${ }^{1,+}$, Sandra Sexton ${ }^{4}$, \\ Leslie Curtin ${ }^{4}$, Joseph A. Spernyak ${ }^{2,5}$, Anurag K. Singh ${ }^{3}$ and Mukund Seshadri 1,2,6,*(D) \\ 1 Laboratory for Translational Imaging, Center for Oral Oncology, Roswell Park Comprehensive Cancer \\ Center, Buffalo, NY 14263, USA; Eftekhar.RajabBolookat@roswellpark.org (E.R.B.); \\ Laurie.Rich@pennmedicine.upenn.edu (L.J.R.) \\ 2 Department of Radiology-Medical Physics Program, University at Buffalo-Jacobs School of Medicine and \\ Biomedical Sciences, 955 Main Street, Buffalo, NY 14203, USA; Harish.Malhotra@roswellpark.org (H.M.); \\ Joseph.Spernyak@roswellpark.org (J.A.S.) \\ 3 Department of Radiation Medicine, Roswell Park Comprehensive Cancer Center, Buffalo, NY 14263, USA; \\ Anurag.Singh@roswellpark.org \\ 4 Laboratory Animal Shared Resource, Roswell Park Comprehensive Cancer Center, Buffalo, NY 14263, USA; \\ Sandra.Sexton@roswellpark.org (S.S.); Leslie.Curtin@roswellpark.org (L.C.) \\ 5 Department of Cell Stress Biology, Roswell Park Comprehensive Cancer Center, Buffalo, NY 14263, USA \\ 6 Department of Dentistry and Maxillofacial Prosthetics, Roswell Park Comprehensive Cancer Center, Buffalo, \\ NY 14263, USA \\ * Correspondence: mukund.seshadri@roswellpark.org \\ + Current affiliation: Center for Magnetic Resonance and Optical Imaging, Department of Radiology, \\ University of Pennsylvania, Philadelphia, PA 19104, USA.
}

Received: 5 February 2020; Accepted: 26 February 2020; Published: 1 March 2020

\begin{abstract}
There is increased interest in the use of magnetic resonance imaging (MRI) for guiding radiation therapy (RT) in the clinical setting. In this regard, preclinical studies can play an important role in understanding the added value of MRI in RT planning. In the present study, we developed and validated a clinically relevant integrated workflow for MRI-guided volumetric arc therapy (VMAT) in a VX2 rabbit neck tumor model of HNSCC. In addition to demonstrating safety and feasibility, we examined the therapeutic impact of MR-guided VMAT using a single high dose to obtain proof-of-concept and compared the response to conventional 2D-RT. Contrast-enhanced MRI (CE-MRI) provided excellent soft tissue contrast for accurate tumor segmentation for VMAT. Notably, MRI-guided RT enabled improved tumor targeting ability and minimal dose to organs at risk (OAR) compared to 2D-RT, which resulted in notable morbidity within a few weeks of RT. Our results highlight the value of integrating MRI into the workflow for VMAT for improved delineation of tumor anatomy and optimal treatment planning. The model combined with the multimodal imaging approach can serve as a valuable platform for the conduct of preclinical RT trials.
\end{abstract}

Keywords: image-guided radiation therapy; VMAT; MRI; HNSCC

\section{Introduction}

Radiation therapy (RT) remains an important component of the standard of care for patients with head and neck squamous cell carcinomas (HNSCC). Clinical studies have shown that volumetric modulated arc therapy (VMAT) can ensure precise radiation delivery to tumors with reduced treatment times while sparing organs at risk (OAR) [1-4]. In VMAT, the radiation beam is continuously reshaped with changing intensity as it moves around the body to ensure highly conformal dose distribution 
to the target tissue while sparing surrounding tissues. While computed tomography (CT) has been traditionally used for radiation treatment planning, there has been increased interest in the use of magnetic resonance imaging (MRI) for guiding RT in the clinical setting [5-7]. In this regard, preclinical studies can play an important role in understanding the added value of MRI for radiation treatment planning and response assessment. Such studies could also assist in the optimization and integration of MRI into the clinical workflow [8,9]

Unfortunately, the vast majority of radiation studies conducted in rodent models do not mimic clinical practice. Experimental studies typically employ crude radiation delivery methods such as orthovoltage $X$-rays or ${ }^{137}$ Cesium irradiators $[10,11]$. These two-dimensional RT (2D-RT) methods lack 3D image guidance for treatment planning and therefore do not allow for precise dose calculations to target volumes or OAR $[12,13]$. This is particularly critical in HNSCC given the abundance of critical structures (e.g., carotid artery) in the head and neck region [14]. The use of 2D-RT methods is not standard for humans but is widely used for preclinical studies in small and large animal models. As a result, the clinical relevance or translational insight gained from these preclinical RT studies remains limited $[8,9,15]$. To address this limitation, image-guided micro irradiators have been recently developed. These commercially available RT platforms enable precision radiotherapy through built-in image guidance for treatment planning [16]. However, these CT-guided radiation research platforms are limited to small animals such as mice and rats [17-19].

While rodent tumor models are cost-effective and widely utilized as preclinical tools to evaluate therapeutic efficacy, their value in predicting clinical activity of novel agents remains controversial. Furthermore, important physiological differences exist between mice and humans that limit the extrapolation of findings from preclinical studies to clinical trials, particularly for RT studies [15]. For example, murine tumors are typically a few millimeters in size compared to human HNSCC that are typically several centimeters in thickness. Similarly, in the head and neck region, differences in salivary gland anatomy between rodents and humans exist that should be considered in the context of RT treatment planning and toxicity assessment [20]. Given these limitations of small animal models, there has been increased interest in the utilization of large animal models of HNSCC for preclinical studies [21-23]. In this regard, we have recently reported on a novel orthotopic rabbit model of HNSCC based on surgical transplantation of Shope cottontail rabbit papilloma virus associated VX2 tumors into the neck of rabbits [24]. Although the VX2 rabbit model has been previously utilized for imaging and therapeutic response studies [25-27], the use of this model for image-guided SBRT or VMAT of head and neck tumors has not been reported.

The overall goal of the present study was to develop and validate a clinically relevant integrated workflow for combined MRI and CT-guided VMAT in the VX2 neck tumor model of HNSCC. In addition to demonstrating safety and feasibility, we examined the therapeutic impact of MR-guided VMAT compared to conventional 2D-RT.

\section{Results}

\subsection{MR-CT and US Imaging of VX2 Neck Tumors-Study Design and Workflow}

The study design and sequence of imaging examinations for the study is shown in Figure 1. Clinical examination provided visual confirmation of tumor growth in all the animals over the three-week period post implantation. Baseline contrast-enhanced MRI (CE-MRI) and non-contrast enhanced CT exams were performed on Day 0 for treatment planning and the developed treatment plan for VMAT was delivered on Day 1. Change in tumor volume evaluated using B-mode US images acquired at baseline, one, two, four, and eight weeks post RT. In addition to radiation delivery, treatment responses and toxicity with IG-VMAT were compared to conventional two-dimensional RT (2D-RT). 


\section{Multimodal Imaging-Guided Radiation Delivery and Response Monitoring}

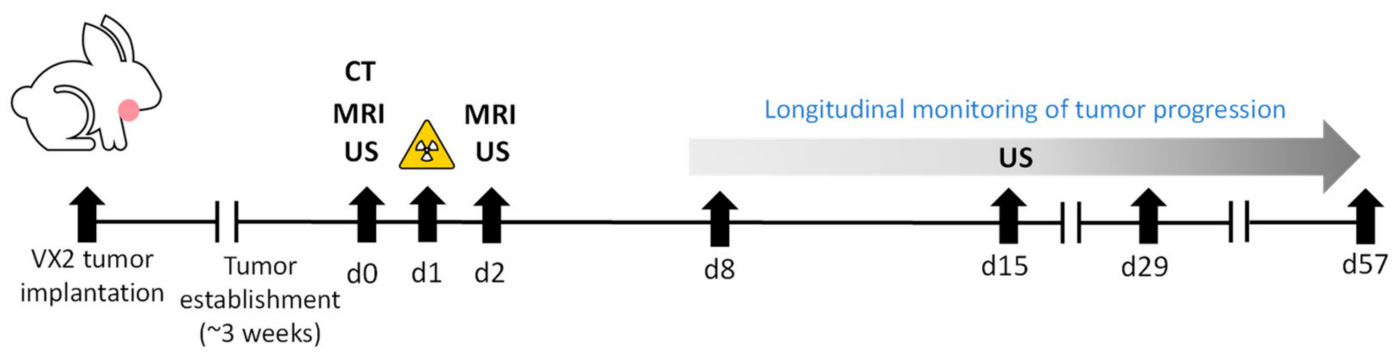

Figure 1. Multimodal imaging-guided radiation delivery and response monitoring. Schematic shows the study design and sequence of imaging examinations conducted in VX2 tumor bearing rabbits. Combined magnetic resonance imaging (MRI) and computed tomography (CT) were used for radiation treatment planning while ultrasound (US) was used to assess changes in tumor growth. Radiation therapy (indicated by the yellow symbol) was delivered $24 \mathrm{~h}$ following baseline line image acquisition.

\subsection{MR-Guided VMAT of VX2 Tumors}

The workflow for MR-guided VMAT of orthotopic VX2 neck tumors is shown in Figure 2. Pretreatment MR images were acquired three weeks post implantation and included T1-weighted acquisitions before and after administration of Gd-DTPA (Magnevist ${ }^{\circledR}$, gadopentetate dimeglumine, Bayer, Whippany, NJ, USA). Post contrast scans showed predominant enhancement in the tumor periphery allowing for clear delineation of tumor boundary (Figure 2D). Following completion of MRI acquisition, animals were allowed to recover for a few hours and subsequently, a treatment planning CT scan was performed (Figure 2A,B and Figure S1). MR images were rigidly co-registered with CT using anatomic landmarks as a guide to ensure prompt treatment planning and radiation delivery within a 24-h period. As evident from the individual images, CE-MRI provided excellent soft tissue contrast to allow for tumor delineation. The tumor was traced on the contrast-enhanced T1-weighted MR images and manually co-registered to the treatment planning CT image using anatomic landmarks as a guide (Figure S2). The segmentation was confirmed on the fused image. Animals in the 2D RT group did not undergo any imaging examination.

\subsection{Treatment Planning and Dosimetry}

The planned target volume (PTV) was defined as $0.5 \mathrm{~cm}$ expansion of the gross tumor volume (GTV). Planning objectives were set to deliver single fraction of 30 Gy to cover at least $95 \%$ of PTV. Organs at risk (OARs) were defined as the skin, trachea, parotid glands, brain, and spinal cord. Dose constraints were set according to Radiation Therapy Oncology Group (RTOG) protocols, which were defined based on single fraction SRS. To check if planning objectives were met, actual dose distributions in PTV and OAR were evaluated by dose volume histograms and 3D isodose lines. If the optimization result was not acceptable, optimization was rerun with modified parameters for priority and dose constraints.

During RT, the rabbits were positioned in relation to the planning CT scan and it was confirmed by use of an on-board cone beam CT (CBCT) using the planning CT set as a reference CT (Figure 3). The $\mathrm{CBCT}$ was overlaid on the reference $\mathrm{CT}$ and necessary corrections to bring the rabbit in desired treatment position by moving the treatment couch. Deviation from the planning CT was set to $2.0 \mathrm{~mm}$ as the tolerance level to reposition and reimage the rabbits. The developed treatment plans for rabbits undergoing MR-guided VMAT fulfilled the requested objective for the target coverage and dose constraints to OAR (Figure S3). As can be seen from the dose distribution and the dose volume histogram (DVH) in Figure 4, the MR-based treatment plan result in homogenous dose delivery to the GTV and reduced dose to tissues outside the PTV. Comparative evaluation of DVH of CT- and MR-based treatment plans showed that tracheal volume receiving $18 \mathrm{~Gy}$ (dose limit) increased from $0 \%$ in the MR-based plan to $85 \%$ in the CT-based plan (Figure S4). 


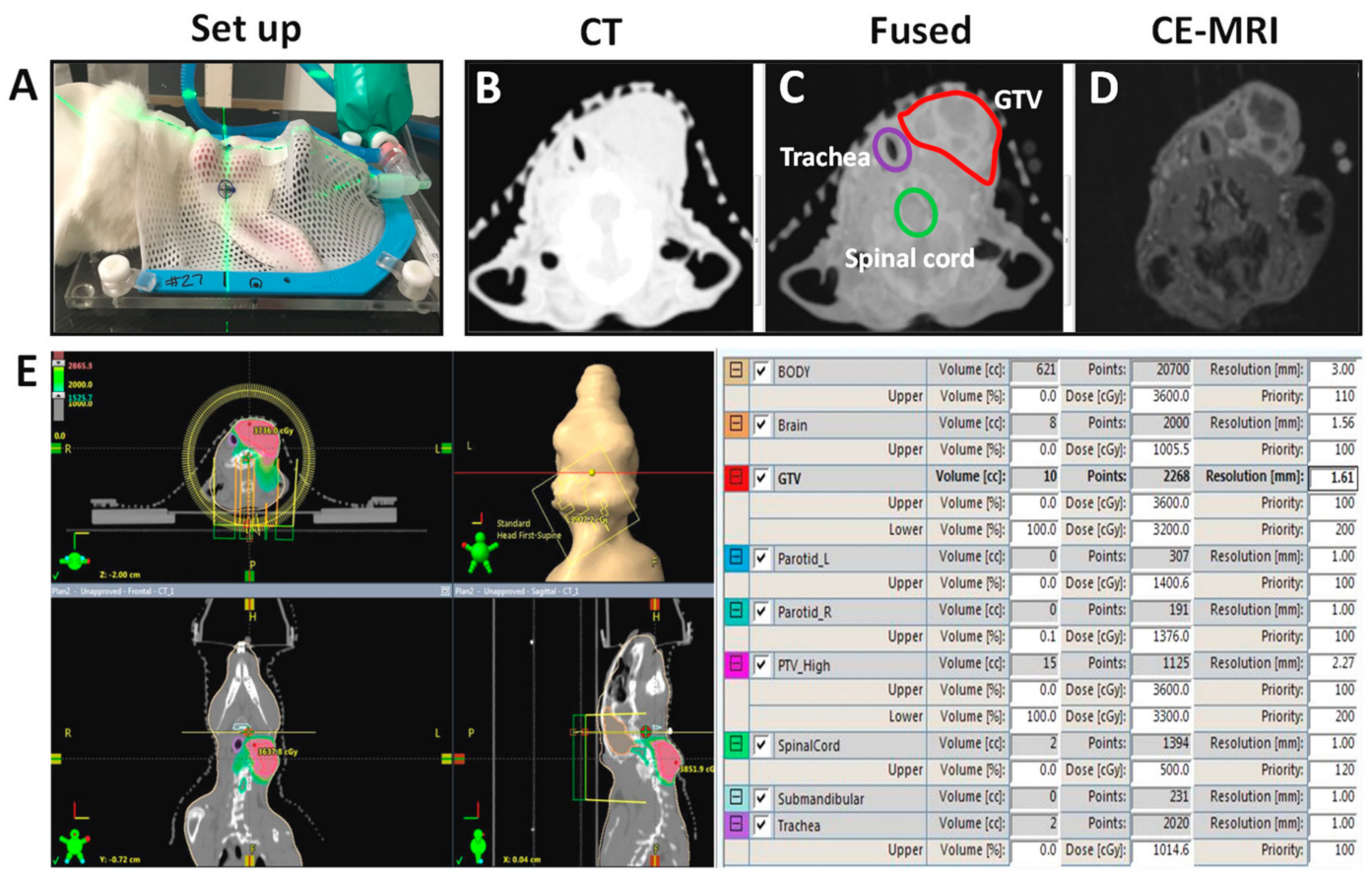

Figure 2. Setup and workflow for MRI-guided VMAT. (A) Setup for CT stimulation of VX2 tumor bearing rabbits. Anesthetized rabbits were positioned on the CT table and a thermoplastic face mask was molded to immobilize the tumor, as shown in the photograph. Axial CT slice (B) and corresponding T1-weighted CE-MRI (D) examinations were performed to enable image-guided VMAT. Images were spatially co-registered (fused) (C) to enable accurate delineation of target volume. Gross tumor volume (GTV) (red) along with OAR including trachea (purple) and spinal cord (green) were traced as shown in the fused image to develop and optimize radiation treatment plans (E). Parameters for plan optimization included upper and lower dose limits for each organ and calculation of dose distribution.

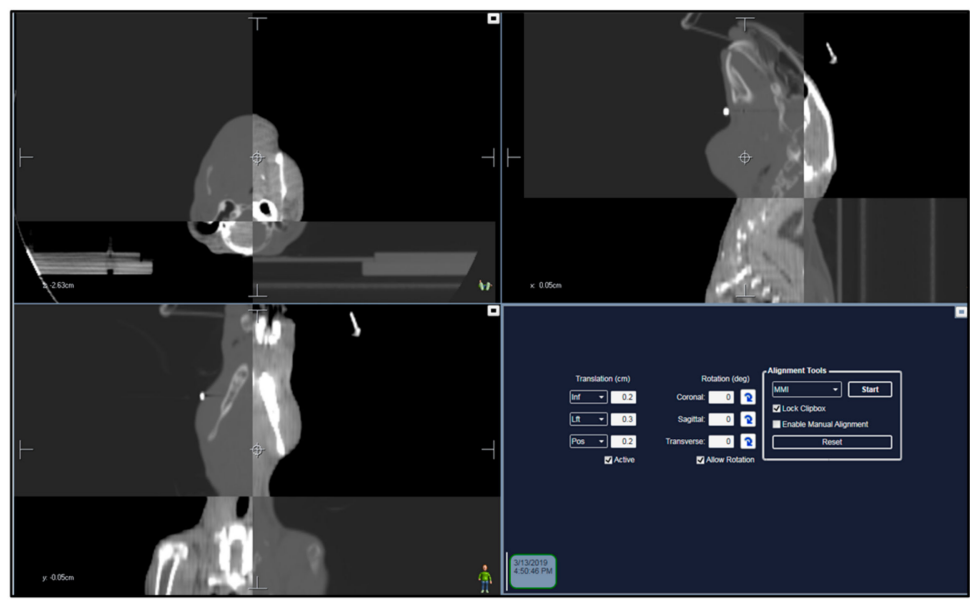

Figure 3. Co-registration of CBCT and treatment planning CT. Panel of images show axial, sagittal, and coronal sections of cone beam CT images aligned with treatment planning CT for verification prior to radiation delivery. Any shifts in position were calculated and applied onto the treatment couch to ensure accurate targeting of the tumor.

At least $95 \%$ of PTV was covered by $30 \mathrm{~Gy}$ in all three rabbits (Rb1, Rb2, and Rb3) treated using VMAT. The volume outside PTV that received $105 \%$ of prescribed dose (31.5 Gy) was zero for all rabbits. Max dose beyond PTV (PTV $+2 \mathrm{~cm}$ ) was 55-56\% of the prescribed dose, which met the protocol limit. Table 1 shows dose constraints for PTV and the dose to OAR obtained by DVH analysis is presented in Table 2. The dose received by OARs was within their tolerance limit. 

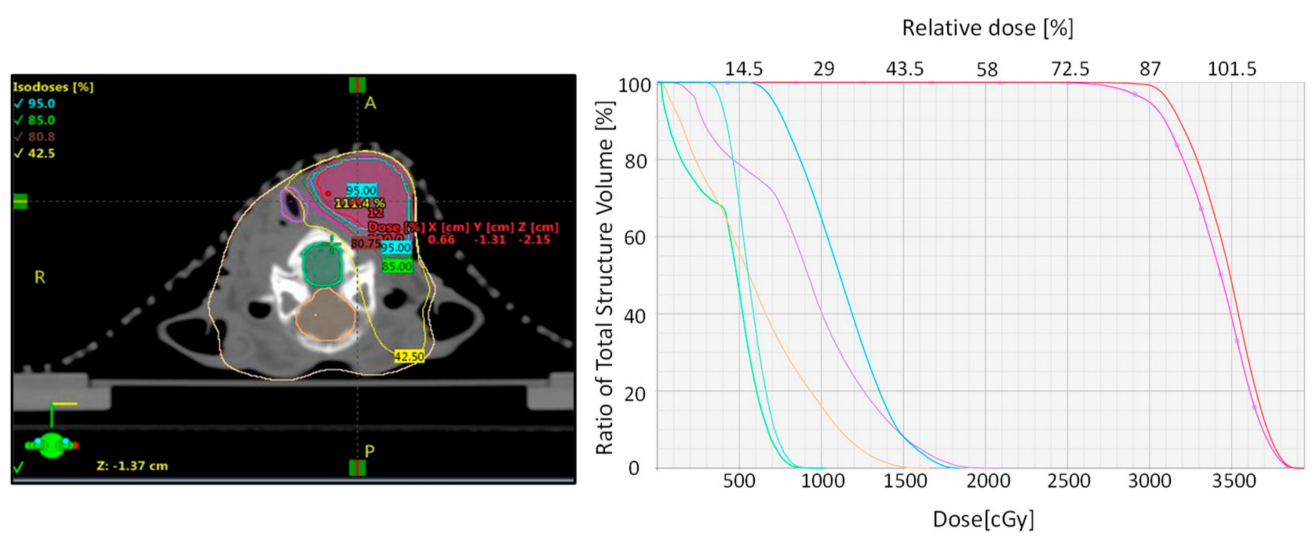

Figure 4. Dose distribution map and dose volume histogram of VX2 tumors. Dose distribution map for tumor volume and OAR displayed as isodoses (\%) is shown on the left. Corresponding dose volume histogram (DVH) for radiation dose to PTV (pink), GTV (red), right and left parotid glands (blue), spinal cord (green), brain (orange), and trachea (purple) is shown on the right.

Table 1. Planning target volume dosimetric parameters generated through DVH analysis (from NRG-BR002 Table 5-5; ClinicalTrials.gov NCT02364557 [28]).

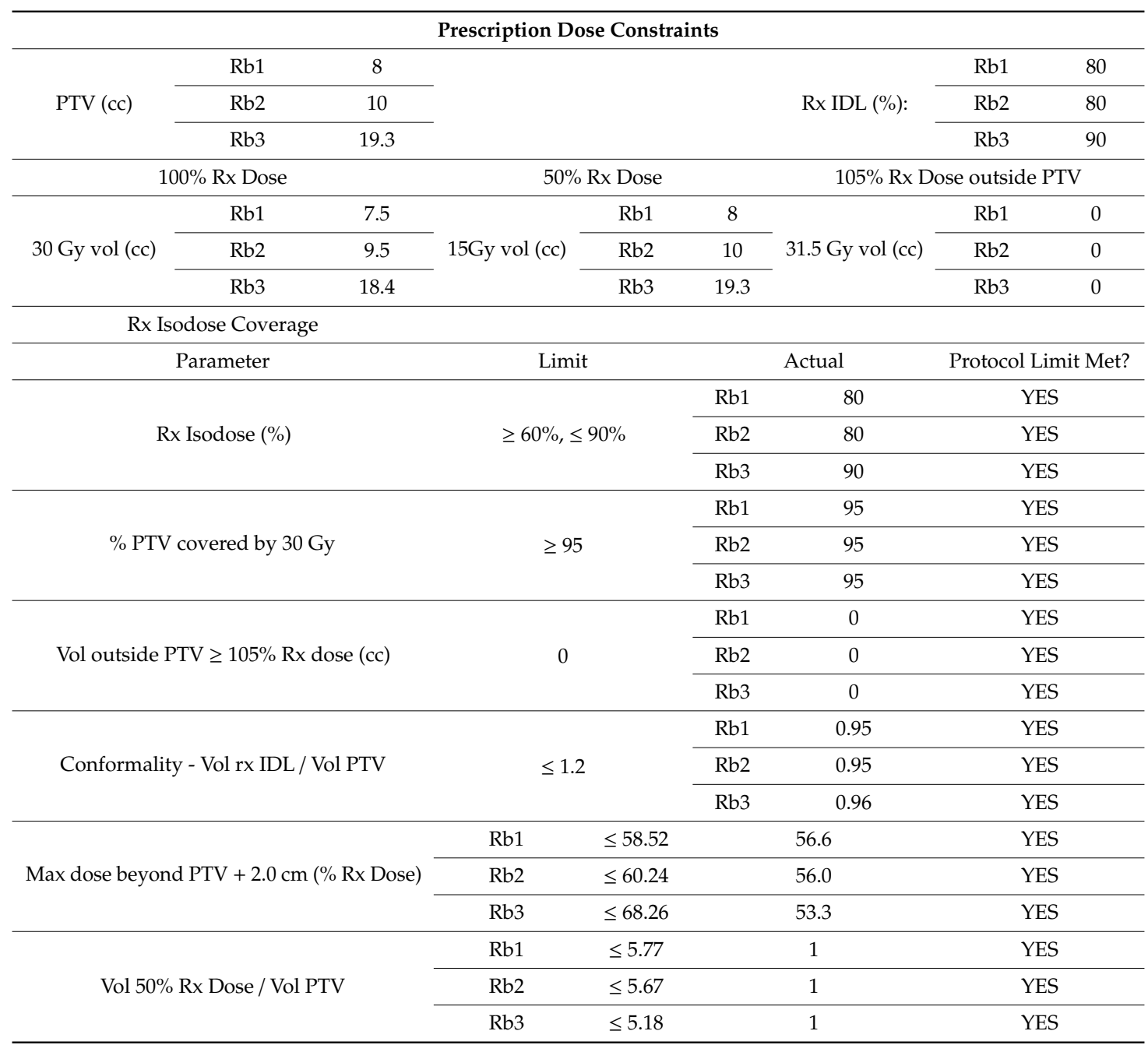


Table 2. Dose Limits on Critical Structures (from NRG-BR002 and RTOG).

\begin{tabular}{|c|c|c|c|c|c|}
\hline \multicolumn{6}{|c|}{ Dose Constraints for OAR. } \\
\hline Organ & Parameter & Limit & & ual & Protocol Limit Met? \\
\hline \multirow{6}{*}{ Spinal Cord } & \multirow{3}{*}{ Volume $>8.0 \mathrm{~Gy}$} & \multirow{3}{*}{$\leq 1.20 \mathrm{cc}$} & $\mathrm{Rb} 1$ & $0.00 \mathrm{cc}$ & Yes \\
\hline & & & $\mathrm{Rb} 2$ & $0.00 \mathrm{cc}$ & Yes \\
\hline & & & $\mathrm{Rb} 3$ & $0.00 \mathrm{cc}$ & Yes \\
\hline & \multirow{3}{*}{ Volume $>10.0$ Gy } & \multirow{3}{*}{$\leq 0.35 \mathrm{cc}$} & $\mathrm{Rb} 1$ & $0.00 \mathrm{cc}$ & Yes \\
\hline & & & $\mathrm{Rb} 2$ & $0.00 \mathrm{cc}$ & Yes \\
\hline & & & $\mathrm{Rb} 3$ & $0.00 \mathrm{cc}$ & Yes \\
\hline \multirow{3}{*}{ Trachea } & \multirow{3}{*}{ Volume $>17.4$ Gy } & \multirow{3}{*}{$\leq 4.00 \mathrm{cC}$} & $\mathrm{Rb} 1$ & $1.00 \mathrm{cc}$ & Yes \\
\hline & & & $\mathrm{Rb} 2$ & $0.00 \mathrm{cc}$ & Yes \\
\hline & & & $\mathrm{Rb} 3$ & $0.00 \mathrm{cc}$ & Yes \\
\hline \multirow{6}{*}{ Skin (surface $-2 \mathrm{~mm}$ ) } & \multirow{3}{*}{ Volume $>25.5$ Gy } & \multirow{3}{*}{$\leq 10.00 \mathrm{cc}$} & $\mathrm{Rb} 1$ & $7.69 \mathrm{cc}$ & Yes \\
\hline & & & $\mathrm{Rb} 2$ & $7.20 \mathrm{cc}$ & Yes \\
\hline & & & $\mathrm{Rb} 3$ & $4.37 \mathrm{cc}$ & Yes \\
\hline & \multirow{3}{*}{ Volume $>27.5$ Gy } & \multirow{3}{*}{$\leq 0.03 \mathrm{cc}$} & $\mathrm{Rb} 1$ & $0.00 \mathrm{cc}$ & Yes \\
\hline & & & $\mathrm{Rb} 2$ & $0.00 \mathrm{cc}$ & Yes \\
\hline & & & $\mathrm{Rb} 3$ & $0.02 \mathrm{cc}$ & Yes \\
\hline \multirow{3}{*}{ Brain } & \multirow{3}{*}{ Volume = $10 \mathrm{~Gy}$} & \multirow{3}{*}{$<10 \mathrm{cc}$} & $\mathrm{Rb} 1$ & $0.00 \mathrm{cc}$ & Yes \\
\hline & & & $\mathrm{Rb} 2$ & $1.50 \mathrm{cc}$ & Yes \\
\hline & & & $\mathrm{Rb3}$ & $2.02 \mathrm{cc}$ & Yes \\
\hline
\end{tabular}

\subsection{Treatment Outcome and Toxicity—MR-Guided VMAT vs. 2D RT}

To examine the therapeutic impact of MR-guided VMAT, longitudinal US was performed over an eight-week observation period. B-mode US was performed and tumor volume measurements were obtained for animals in control, MR-guided VMAT, and 2D-RT groups (Figure 5).
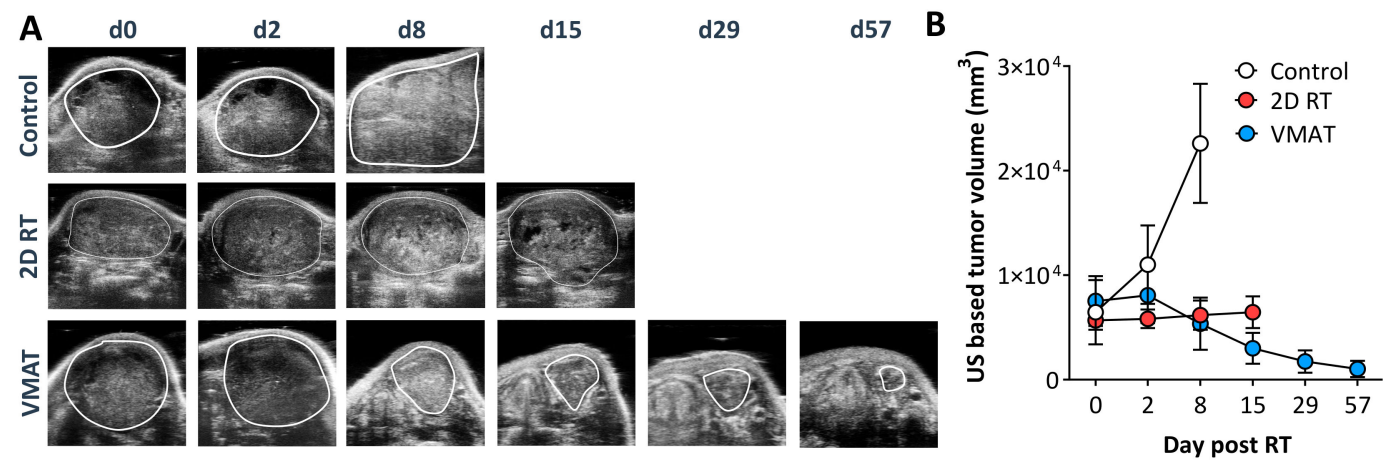

Figure 5. US-based monitoring of VX2 response to MR-guided VMAT. (A) Longitudinal B-mode US images of a representative animal from control, VMAT, and 2D RT groups. Tumor is outlined in white on all the images. Columns represent days post RT (d, days) (B) Corresponding tumor volume measurements at different time points post RT in VMAT, 2D-RT, and control cohorts. Animals treated by VMAT showed $85-100 \%$ reduction in tumor volume by Day 57 . However, animals in 2D-RT cohort demonstrated steady increase in tumor volume and they were euthanized 14 days post RT due to significant morbidity.

Good concordance was seen between tumor volume measurements obtained with US and MRI (Figure S5). Figure 5A shows longitudinal US images of a single animal in each group over the 
eight-week period. Corresponding tumor volume curves are shown in Figure 5B. Control animals showed a rapid increase in tumor volume within 10 days that was associated with considerable weight loss necessitating euthanasia. Tumor bearing rabbits treated with 2D RT using the orthovoltage radiation system showed growth inhibition. However, animals had to be euthanized at two weeks post RT due considerable morbidity (weight loss, poor appetite, and radiation dermatitis). In comparison, animals that underwent VMAT showed an initial tumor growth inhibition (one week post RT), which was followed by a marked reduction in tumor volume ( $85-100 \%$ reduction at eight weeks post RT).

\section{Discussion}

The overall goal of radiation therapy is to safely and accurately deliver appropriate radiation dose to the tumor while minimizing the dose to surrounding normal tissues. In the present study, we established a preclinical protocol that closely mimics clinical workflow for combined MR and CT to enable accurate tumor delineation and dosimetry in a VX2 rabbit neck tumor model of HNSCC. Our results demonstrate the feasibility and therapeutic impact of IG-VMAT compare to 2D RT. The model combined with the multimodal imaging approach can serve as a valuable platform for the conduct of preclinical RT trials.

The field of radiation medicine has experienced tremendous advancements over the last two decades including the development of state-of-the-art CT scanners for 3D imaging and powerful treatment planning systems [29]. Improved radiation delivery techniques such as IMRT and VMAT with image guidance are being increasingly evaluated in the clinical setting for patients with solid tumors including HNSCC [2-4,30-32]. Unfortunately, image-guided RT delivery methods are not routinely employed in preclinical studies. Studies in rodent tumor models have typically utilized orthovoltage systems that are susceptible to dosimetry errors [12,13]. Commercial CT-guided RT platforms have been recently developed and are being increasingly utilized for studies in small animal models of cancer [16-19]. While mouse models are widely utilized in research, the clinical or translational value of radiation studies in murine systems remains unclear. As a result, there has been increased interest in developing and applying canine (dogs), feline (cats), and leporine (rabbits) models of cancer for preclinical research [21-27]. However, the literature on the implementation of IG-VMAT in large animal models of cancer, especially HNSCC, is limited.

Since commercially available image-guided RT systems are not amenable to conducting studies using large animals, we developed and refined a protocol based on MR-guided VMAT in rabbits bearing VX2 tumors in the neck. The excellent soft tissue contrast afforded by MRI allowed for precise tumor delineation while the linear accelerator with onboard image guidance enabled precise delivery to the target volume. Despite the close association of the tumor to critical structures in the neck such as the trachea, spinal cord, and brain, a single high dose of $30 \mathrm{~Gy}$ was safely delivered under MR-guidance and resulted in a marked reduction in tumor volume. In recent years, highly conformal VMAT can be potentially delivered using a hypofractionated regimen for patients with recurrent disease following fractionated radiation [33]. We therefore evaluated our MR-guided RT approach using a single high dose to obtain proof-of-concept. While MR-guided RT has been reported in rat models of prostate, pancreas, and brain tumors [34-36], to the best of our knowledge, the potential of this approach has not been previously demonstrated in HNSCC. Our results also highlight the superior targeting ability of VMAT with minimal dose to OAR. In contrast, 2D-RT did not allow adequate sparing of these tissues and resulted in notable morbidity within a few weeks of RT. Consistent with our observations and these clinical studies, Wang et al. reported the potential of VMAT and simultaneous integrated boost technique is feasible in the rabbit VX2 limb tumor model [37]. Similarly, Dolera et al. reported on the safety of VMAT for treating rabbit thymomas as well as brain tumors in pets [38].

Our study limitations warrant recognition. Since our main goal was to establish and validate the feasibility of a clinically-relevant workflow, the sample size for our cohorts was relatively small. While we were able to achieve good co-registration to enable MR-guided treatment planning, we did 
not investigate the potential geometric distortions among the MR, CT and CBCT images. Future studies should evaluate and correct for these potential distortions to further improve the accuracy of MR-guided RT [35]. Second, our study was focused on evaluation of primary tumor response to RT. It would be clinically insightful to examine the long-term survival along with evaluation of disease recurrence or metastases with MR-guided VMAT. As such, integrating MR into the clinical RT workflow is being actively investigated in the clinical setting. Our platform could also be valuable in the conduct of preclinical trials to evaluate and optimize the combination of RT with targeted agents and immunotherapy.

\section{Materials and Methods}

\subsection{Animals}

Adult male, specific-pathogen free, New Zealand White (NZW) rabbits (body weight range, 1.4-1.8 kg) were purchased from Charles River Corporation (Saint Constant, Quebec, Canada). Animals were acclimatized for one week before experiment. Animals were housed in individual stainless-steel cages containing noncontact bedding $\left(24^{\prime \prime} \mathrm{w} \times 24^{\prime \prime} \mathrm{d} \times 16^{\prime \prime} \mathrm{h}\right)$ under 12-h light and dark cycles. Rabbits were fed high-fiber diet (Rabbit diet \# 2031; Harlan Teklad, KY, USA) and purified water and dietary enrichment consisted of Timothy Hay cubes and kale. Environmental enrichment in the form of manipulanda (Bio-Serv, Flemington, NY, USA) was also provided.

\subsection{Surgical Procedure for Establishing VX2 Tumors in the Neck}

We recently described the surgical procedure for establishing VX2 tumors in the neck of rabbits [24]. Prior to the surgical procedure, topical lidocaine and prilocaine anesthetic cream (Hi Tech Pharmacal Co. Inc., Amityville, NY, USA) was applied to the ear. Animals were sedated using acepromazine maleate $(0.3 \mathrm{mg} / \mathrm{kg}$, i.v. Phoenix Pharm Inc., Burlingame, CA, USA). Induction and maintenance of anesthesia were performed using inhalational isoflurane (Patterson Logistics Services Inc., Mount Joy, PA, USA). Preoperative analgesia was achieved by intramuscular administration of buprenorphine hydrochloride $(0.05 \mathrm{mg} / \mathrm{kg}$; Patterson Logistics Services Inc., Mount Joy, PA, USA). An incision was made on the skin along the length of the neck followed by blunt dissection of subcutaneous tissue and fascia. A piece of sterile VX2 tumor tissue (approximately $2 \mathrm{~mm} \times 2 \mathrm{~mm}$ ) from a donor rabbit was placed within a pocket created within the lateral aspect of the sternohyoid muscle tissue under anesthesia. The pocket was closed with a 6-0 Vicryl suture and the skin wound closed with tissue glue and ethilon suture. All surgical procedures were performed under aseptic conditions in an AAALAC-accredited facility and in accordance with protocols approved by the Institutional Animal Care and Use Committee (Rb 1285; Approval date: 29 January 2015). Following surgical implantation, animals were monitored daily for general health, signs of distress, and/or tumor growth. Humane end points for euthanasia included tumor size greater than $4 \mathrm{~cm}$ in diameter and/or any signs of distress.

\subsection{Magnetic Resonance Imaging}

MR imaging examinations were performed using a 4.7T/33 cm horizontal bore magnet (GE NMR Instruments, Fremont, CA, USA) incorporating AVANCE digital electronics (Bruker Biospec with Paravision 3.0.2; Bruker Biospin., Billerica, MA, USA). Animals were sedated using acepromazine maleate followed by inhalational anesthesia using Isoflurane (Benson Medical Industries, Markham, ON, Canada). A catheter was placed into the marginal auricular vein for administration of contrast. The rabbits were secured in a form-fitted, MR compatible cradle in the supine position along with a phantom (1\% agarose with NMR tube). T1-weighted, 3D spoiled gradient recalled echo (SPGR) scans were performed with the following parameters: $\mathrm{TR}=15 \mathrm{~ms}$; $\mathrm{TE}=3 \mathrm{~ms}$; flip angle $=25^{\circ}$; field of view = $16 \mathrm{~cm} \times 12 \mathrm{~cm} \times 12 \mathrm{~cm}$; matrix size = $192 \times 128 \times 128$; number of averages $(\mathrm{NEX})=2$; and bandwidth $=83.3 \mathrm{kHz}$. Images were obtained before and up to $8 \mathrm{~min}$ post-gadolinium injection (Magnevist: $0.05 \mathrm{mmol} / \mathrm{kg}$, flush $10 \mathrm{~mL}$ saline after injection administered intravenously). Following MR image 
acquisition, raw image sets were transferred to a processing workstation and converted into Analyze $\mathrm{AM}^{\mathrm{TM}}$ format (Analyze 7.0; AnalyzeDirect, Overland Park, KS, USA). Image segmentation included a region of interest traced around the entire tumor area excluding the surrounding skin.

\subsection{Radiation Therapy}

Ten rabbits were used for experimental studies. Three weeks post implantation, rabbits were assigned to control $(n=4)$, MR-guided VMAT $(n=3)$, or 2D-RT $(n=3)$ groups. For VMAT, rabbits were anesthetized (isoflurane, $2 \%$ ) and placed in a supine position in a Big Bore CT scanner (GE discovery RT 16 slices, slice thickness: $0.125 \mathrm{~cm}, 120 \mathrm{kV}, 99 \mathrm{~mA}$, FOV $35 \mathrm{~cm}$, matrix size $512 \times 512$ ). CT images were imported into the Eclipse treatment planning system (version 13.6, Varian Medical Systems, Palo Alto, CA, USA). MRI and CT scans were fused manually using anatomic landmarks as a guide through the image registration algorithm provided in the treatment planning system. Two full arcs were designed in clockwise rotation $\left(181^{\circ}\right.$ to $\left.179^{\circ}\right)$ and in counter-clockwise rotation $\left(179^{\circ}\right.$ to $\left.181^{\circ}\right)$. The collimator angles were $30^{\circ}$ and $330^{\circ}$ for the first and second arc, respectively. Anisotropic Analytical Algorithm (AAA) with a calculation grid set to $2.5 \mathrm{~mm}$ was used for dose calculation with heterogeneity correction. RT was applied with $6 \mathrm{MV}$ photon beams and maximum dose rate of $600 \mathrm{MU} / \mathrm{min}$ using Varian linear accelerator (Triology, Varian Medical Systems, Palo Alto, CA, USA) with the Millennium 120-mutileaf collimator. Conventional RT of the prescribed dose (30 Gy calculated by a single point along the central axis) was delivered by two opposite lateral fields using the Philips RT 250 Orthovoltage X-ray unit (Philips Medical Systems, Andover, MA, USA) equipped with an aluminum filter and operating at $250 \mathrm{kV} / 17.7 \mathrm{~mA}$. The dose rate at this setting was $\sim 0.68 \mathrm{~Gy} / \mathrm{min}$. Animals were monitored with a closed-circuit TV during irradiation and observed after completion of treatment to ensure full recovery.

\subsection{Ultrasound}

Experimental US examination was performed using a commercially available high-frequency ultrasound system (FujiFilm VisualSonics Inc., Toronto, Canada) consisting of a $15 \mathrm{MHz}$ transducer synchronized to a micro-ultrasound system and a work station to process and reconstruct the images. Animals were positioned on their back for imaging and three-dimensional (3D) B-mode US images were acquired to calculate tumor volume. Animal vitals were monitored continuously during the imaging procedure and following completion of imaging to ensure full recovery.

\section{Conclusions}

We demonstrated the ability of MRI-guided RT in a large animal model of HNSCC. Our results highlight the value of integrating MRI into the workflow for VMAT for improved delineation of tumor anatomy and optimal treatment planning. Our clinically relevant model system and workflow could enable future evaluation of adaptive planning based on functional MRI methods such as blood oxygenation level dependent MRI (BOLD-MRI) and tissue oxygenation level dependent MRI (TOLD-MRI).

Supplementary Materials: The following are available online at http://www.mdpi.com/2072-6694/12/3/572/s1, Figure S1: Rabbit set up and treatment planning, Figure S2: Registration of CT and MRI on treatment planning system, Figure S3: Treatment planning interface, Figure S4: Dose volume histogram of CT-based treatment plans, Figure S5: Correlation between MR- and US-based tumor volume.

Author Contributions: Conceptualization, E.R.B., H.M., L.J.R., A.K.S., and M.S.; Methodology, all authors; Formal analysis, E.R.B., H.M., and L.J.R.; Investigation, E.R.B., H.M., L.J.R., A.K.S. and M.S.; Resources, A.K.S. and M.S.; Data curation, E.R.B., H.M., L.J.R., and J.A.S.; Writing—original draft preparation, E.R.B., L.J.R., and M.S.; Writing-review and editing, all authors; Visualization, E.R.B. and L.J.R.; Supervision, H.M., A.K.S., and M.S.; Project administration, A.K.S. and M.S.; and Funding acquisition, M.S. All authors have read and agreed to the published version of the manuscript.

Funding: This work was supported by grants from the National Cancer Institute 1R01CA204636, P30CA06156, and the Roswell Park Alliance Foundation. 
Acknowledgments: The authors would like thank Ms. Jennifer Buil for assistance with CT simulation and radiation studies and the staff from the shared resources at Roswell Park Comprehensive Cancer Center for their technical assistance in performing the experiments.

Conflicts of Interest: The authors declare no conflict of interest. The funders had no role in the design of the study; in the collection, analyses, or interpretation of data; in the writing of the manuscript, or in the decision to publish the results.

\section{References}

1. Vanetti, E.; Clivio, A.; Nicolini, G.; Fogliata, A.; Ghosh-Laskar, S.; Agarwal, J.P.; Upreti, R.R.; Budrukkar, A.; Murthy, V.; Deshpande, D.D.; et al. Volumetric modulated arc radiotherapy for carcinomas of the oro-pharynx, hypo-pharynx and larynx: A treatment planning comparison with fixed field IMRT. Radiother. Oncol. 2009, 92, 111-117. [CrossRef]

2. Verbakel, W.F.; Cuijpers, J.P.; Hoffmans, D.; Bieker, M.; Slotman, B.J.; Senan, S. Volumetric intensity-modulated arc therapy vs. conventional IMRT in head-and-neck cancer: A comparative planning and dosimetric study. Int. J. Radiat. Oncol. Biol. Phys. 2009, 74, 252-259. [CrossRef] [PubMed]

3. Syam Kumar, S.A.; Vivekanandan, N.; Sriram, P. A study on conventional IMRT and RapidArc treatment planning techniques for head and neck cancers. Rep. Pract. Oncol. Radiother. 2012, 17, 168-752. [CrossRef] [PubMed]

4. Franzese, C.; Fogliata, A.; Clerici, E.; Franceschini, D.; Villa, E.; D'Agostino, G.; Navarria, P.; Mancosu, P.; Tomatis, S.; Cozzi, L.; et al. Toxicity profile and early clinical outcome for advanced head and neck cancer patients treated with simultaneous integrated boost and volumetric modulated arc therapy. Radiat. Oncol. 2015, 10, 224. [CrossRef] [PubMed]

5. Chen, A.M.; Cao, M.; Hsu, S.; Lamb, J.; Mikaeilian, A.; Yang, Y.; Agazaryan, N.; Low, D.A.; Steinberg, M.L. Magnetic resonance imaging guided reirradiation of recurrent and second primary head and neck cancer. Adv. Radiat. Oncol. 2017, 2, 167-175. [CrossRef] [PubMed]

6. Chen, A.M.; Hsu, S.; Lamb, J.; Yang, Y.; Agazaryan, N.; Steinberg, M.L.; Low, D.A.; Cao, M. MRI-guided radiotherapy for head and neck cancer: Initial clinical experience. Clin. Transl. Oncol. 2018, 20, 160-168. [CrossRef] [PubMed]

7. Kiser, K.; Meheissen, M.A.M.; Mohamed, A.S.R.; Kamal, M.; Ng, S.P.; Elhalawani, H.; Jethanandani, A.; He, R.; Ding, Y.; Rostom, Y.; et al. Prospective quantitative quality assurance and deformation estimation of MRI-CT image registration in simulation of head and neck radiotherapy patients. Clin. Transl. Radiat. Oncol. 2019, 18, 120-127. [CrossRef]

8. Tillner, F.; Thute, P.; Butof, R.; Krause, M.; Enghardt, W. Pre-clinical research in small animals using radiotherapy technology-A bidirectional translational approach. Z. Med. Phys. 2014, 24, 335-351. [CrossRef]

9. Ghita, M.; Brown, K.H.; Kelada, O.J.; Graves, E.E.; Butterworth, K.T. Integrating Small Animal Irradiators with Functional Imaging for Advanced Preclinical Radiotherapy Research. Cancers 2019, 11, 170. [CrossRef]

10. Medina, L.A.; Herrera-Penilla, B.I.; Castro-Morales, M.A.; García-López, P.; Jurado, R.; Pérez-Cárdenas, E.; Chanona-Vilchis, J.; Brandan, M.E. Use of an orthovoltage $X$-ray treatment unit as a radiation research system in a small-animal cancer model. J. Exp. Clin. Cancer Res. 2008, 27, 57. [CrossRef] [PubMed]

11. O'Steen, S.; Green, D.J.; Gopal, A.K.; Orozco, J.J.; Kenoyer, A.L.; Lin, Y.; Wilbur, D.S.; Hamlin, D.K.; Fisher, D.R.; Hylarides, M.D.; et al. Venetoclax Synergizes with Radiotherapy for Treatment of B-cell Lymphomas. Cancer Res. 2017, 77, 3885-3893. [CrossRef] [PubMed]

12. Yoshizumi, T.; Brady, S.L.; Robbins, M.E.; Bourland, J.D. Specific issues in small animal dosimetry and irradiator calibration. Int. J. Radiat. Biol. 2011, 87, 1001-1010. [CrossRef] [PubMed]

13. Chen, Q.; Molloy, J.; Izumi, T.; Sterpin, E. Impact of backscatter material thickness on the depth dose of orthovoltage irradiators for radiobiology research. Phys. Med. Biol. 2019, 64, 055001. [CrossRef] [PubMed]

14. Gebhardt, B.J.; Vargo, J.A.; Ling, D.; Jones, B.; Mohney, M.; Clump, D.A.; Ohr, J.P.; Ferris, R.L.; Heron, D.E. Carotid Dosimetry and the Risk of Carotid Blowout Syndrome After Reirradiation With Head and Neck Stereotactic Body Radiation Therapy. Int. J. Radiat. Oncol. Biol. Phys. 2018, 101, 195-200. [CrossRef] [PubMed]

15. Koontz, B.F.; Verhaegen, F.; De Ruysscher, D. Tumour and normal tissue radiobiology in mouse models: How close are mice to mini-humans? Br. J. Radiol. 2017, 90, 20160441. [CrossRef] [PubMed] 
16. Verhaegen, F.; Granton, P.; Tryggestad, E. Small animal radiotherapy research platforms. Phys. MedBiol. 2011, 56, R55-R83. [CrossRef]

17. Wong, J.; Armour, E.; Kazanzides, P.; Iordachita, I.; Tryggestad, E.; Deng, H.; Matinfar, M.; Kennedy, C.; Liu, Z.; Chan, T.; et al. High-resolution, small animal radiation research platform with x-ray tomographic guidance capabilities. Int. J. Radiat. Oncol. Biol. Phys. 2008, 71, 1591-1599. [CrossRef]

18. Grover, A.R.; Kimler, B.F.; Duncan, F.E. Use of a Small Animal Radiation Research Platform (SARRP) facilitates analysis of systemic versus targeted radiation effects in the mouse ovary. J. Ovarian Res. 2018, 11, 72. [CrossRef]

19. Gutierrez, S.; Descamps, B.; Vanhove, C. MRI-Only Based Radiotherapy Treatment Planning for the Rat Brain on a Small Animal Radiation Research Platform (SARRP). PLoS ONE 2015, 10, e0143821. [CrossRef]

20. Maruyama, C.L.; Monroe, M.M.; Hunt, J.P.; Buchmann, L.; Baker, O.J. Comparing human and mouse salivary glands: A practice guide for salivary researchers. Oral Dis. 2019, 25, 403-415. [CrossRef]

21. Supsavhad, W.; Dirksen, W.P.; Martin, C.K.; Rosol, T.J. Animal models of head and neck squamous cell carcinoma. Vet. J. 2016, 210, 7-16. [CrossRef] [PubMed]

22. Liu, D.; Xiong, H.; Ellis, A.E.; Northrup, N.C.; Dobbin, K.K.; Shin, D.M.; Zhao, S. Canine spontaneous head and neck squamous cell carcinomas represent their human counterparts at the molecular level. PLoS Genet. 2015, 11, e1005277. [CrossRef] [PubMed]

23. Oak, C.; Ahn, Y.C.; Nam, S.J.; Jung, M.H.; Hwang, S.S.; Chae, Y.G.; Lee, H.S.; Lee, K.D.; Jung, M.J.; Chun, B.K.; et al. Multimodal imaging using optical coherence tomography and endolaryngeal ultrasonography in a new rabbit VX2 laryngeal cancer model. Lasers Surg. Med. 2015, 47, 704-710. [CrossRef] [PubMed]

24. Rich, L.J.; Sexton, S.; Curtin, L.; Seshadri, M. Spatiotemporal Optoacoustic Mapping of Tumor Hemodynamics in a Clinically Relevant Orthotopic Rabbit Model of Head and Neck Cancer. Transl. Oncol. 2017, 10, 839-845. [CrossRef]

25. Lijowski, M.; Caruthers, S.; Hu, G.; Zhang, H.; Scott, M.J.; Williams, T.; Erpelding, T.; Schmieder, A.H.; Kiefer, G.; Gulyas, G.; et al. High sensitivity: High-resolution SPECT-CT/MR molecular imaging of angiogenesis in the Vx2 model. Investig. Radiol. 2009, 44, 15-22. [CrossRef]

26. Zhang, L.; Liu, F.Y.; Fu, J.X.; Duan, F.; Fan, Q.S.; Wang, M.Q. Hepatic arterial administration of sorafenib and iodized oil effectively attenuates tumor growth and intrahepatic metastasis in rabbit VX2 hepatocellular carcinoma model. Int. J. Clin. Exp. Pathol. 2014, 7, 7775-7781.

27. Lam, M.K.; Oerlemans, C.; Froeling, M.; Deckers, R.; Barten-Van Rijbroek, A.D.; Viergever, M.A.; Moonen, C.T.; Bos, C.; Bartels, L.W. DCE-MRI and IVIM-MRI of rabbit Vx2 tumors treated with MR-HIFU-induced mild hyperthermia. J. Ther. Ultrasound 2016, 4, 9. [CrossRef] [PubMed]

28. ClinicalTrials.gov NCT02364557. Available online: https://clinicaltrials.gov/ct2/show/NCT02364557 (accessed on 1 March 2020).

29. Saw, C.B.; Li, S. 3D treatment planning systems. Med. Dosim. 2018, 43, 103-105. [CrossRef]

30. Teoh, M.; Clark, C.H.; Wood, K.; Whitaker, S.; Nisbet, A. Volumetric modulated arc therapy: A review of current literature and clinical use in practice. Br. J. Radiol. 2011, 84, 967-996. [CrossRef]

31. Brown, M.L.; Glanzmann, C.; Huber, G.; Bredell, M.; Rordorf, T.; Studer, G. IMRT/VMAT for malignancies in the head-and-neck region: Outcome in patients aged 80. Strahlenther. Onkol. 2016, 192, 526-536. [CrossRef]

32. Fung-Kee-Fung, S.D.; Hackett, R.; Hales, L.; Warren, G.; Singh, A.K. A prospective trial of volumetric intensity-modulated arc therapy vs. conventional intensity modulated radiation therapy in advanced head and neck cancer. World J. Clin. Oncol. 2012, 3, 57-62. [CrossRef] [PubMed]

33. Bourgeois, D.J., 3rd.; Singh, A.K. Single-fraction stereotactic body radiation therapy for sinonasal malignant melanoma. Head Neck 2015, 37, E34-E37. [CrossRef] [PubMed]

34. Bolcaen, J.; Descamps, B.; Deblaere, K.; Boterberg, T.; Hallaert, G.; Van den Broecke, C.; Decrock, E.; Vral, A.; Leybaert, L.; Vanhove, C.; et al. MRI-guided 3D conformal arc micro-irradiation of a F98 glioblastoma rat model using the Small Animal Radiation Research Platform (SARRP). J. Neurooncol. 2014, 120, 257-266. [CrossRef] [PubMed]

35. Chiu, T.D.; Arai, T.J.; Campbell, I.J.; Jiang, S.B.; Mason, R.P.; Stojadinovic, S. MR-CBCT image-guided system for radiotherapy of orthotopic rat prostate tumors. PLoS ONE 2018, 13, e0198065. [CrossRef]

36. Dobiasch, S.; Kampfer, S.; Habermehl, D.; Duma, M.N.; Felix, K.; Strauss, A.; Schilling, D.; Wilkens, J.J.; Combs, S.E. MRI-based high-precision irradiation in an orthotopic pancreatic tumor mouse model: A treatment planning study. Strahlenther. Onkol. 2018, 194, 944-952. [CrossRef] 
37. Wang, C.W.; Zhou, Y.; Bai, J.P.; Liu, H.; Liu, Y.; Shi, G.L.; Ding, J.J.; Ma, D.H.; Li, W.T.; Xie, P.M.; et al. Application of Volumetric Modulated Arc Therapy and Simultaneous Integrated Boost Techniques to Prepare "Safe Margin" in the Rabbit VX2 Limb Tumor Model. Med. Sci. Monit. 2015, 21, 2397-2405. [CrossRef]

38. Dolera, M.; Malfassi, L.; Mazza, G.; Urso, G.; Sala, M.; Marcarini, S.; Carrara, N.; Pavesi, S.; Finesso, S.; Kent, M.S. Feasibility for Using Hypofractionated Stereotactic Volumetric Modulated Arc Radiotherapy (Vmat) with Adaptive Planning for Treatment of Thymoma in Rabbits: 15 Cases. Vet. Radiol. Ultrasound 2016, 57, 313-320. [CrossRef]

(C) 2020 by the authors. Licensee MDPI, Basel, Switzerland. This article is an open access article distributed under the terms and conditions of the Creative Commons Attribution (CC BY) license (http://creativecommons.org/licenses/by/4.0/). 